\title{
DIFFERENTIATION OF SOME FUNCTIONALS OF RISK PROCESSES, AND OPTIMAL RESERVE ALLOCATION
}

\author{
STÉPHANE LOISEL, * Université Lyon 1
}

\begin{abstract}
For general risk processes, we introduce and study the expected time-integrated negative part of the process on a fixed time interval. Differentiation theorems are stated and proved. They make it possible to derive the expected value of this risk measure, and to link it with the average total time below 0 , studied by Dos Reis, and the probability of ruin. We carry out differentiation of other functionals of one-dimensional and multidimensional risk processes with respect to the initial reserve level. Applications to ruin theory, and to the determination of the optimal allocation of the global initial reserve that minimizes one of these risk measures, illustrate the variety of fields of application and the benefits deriving from an efficient and effective use of such tools.
\end{abstract}

Keywords: Ruin theory; sample path property; optimal reserve allocation; multidimensional risk process; risk measure

2000 Mathematics Subject Classification: Primary 60G17

Secondary 60G55; 91B30; 91B32; 62P05

\section{Introduction}

Consider the one-dimensional risk process $R_{t}=u+X_{t}$, representing the surplus of an insurance company at time $t$, with initial reserve $u$ and with $X_{t}=c t-S_{t}$, where $c>0$ is the premium income rate and $S_{t}$ is, in the most classical case, a compound Poisson process (although we do not limit ourselves to the Poisson case here). For such processes, many risk measures have been considered (see, for example, Gerber (1988), Dufresne and Gerber (1988), and Picard (1994)): the time to ruin $T_{u}=\inf \left\{t>0: u+X_{t}<0\right\}$; the severity of ruin $u+X_{T_{u}}$; the pair $\left(T_{u}, u+X_{T_{u}}\right)$; the time in the red (that is, below 0) $T_{u}^{\prime}-T_{u}$ from the time of first ruin to the time of first recovery, where $T_{u}^{\prime}=\inf \left\{t>T_{u}: u+X_{t}=0\right\}$; the maximal ruin severity $\inf _{t>0}\left(u+X_{t}\right)$; the aggregate severity of ruin until recovery $J(u)=\int_{T_{u}}^{T_{u}^{\prime}}\left|u+X_{t}\right| \mathrm{d} t$; etc. Dos Reis (1993) studied the total time in the red $\tau(u)=\int_{0}^{\infty} \mathbf{1}_{\left\{u+X_{t}<0\right\}} \mathrm{d} t$, using results of Gerber (1988).

These random variables are all drawn from infinite-time ruin theory, or involve the behaviour of the risk process between ruin times and recovery times. It seems interesting to consider risk measures based on some fixed time interval $[0, T]$ ( $T$ may be infinite). One of the simplest penalty functions might be the expected value of the time-aggregated negative part of the risk process:

$$
\mathrm{E}\left(I_{T}\right):=\mathrm{E}\left(\int_{0}^{T} \mathbf{1}_{\left\{R_{t}<0\right\}}\left|R_{t}\right| \mathrm{d} t\right)
$$

Received 2 June 2004; revision received 29 September 2004.

* Postal address: Ecole ISFA, 50 avenue Tony Garnier, 69366 Lyon Cedex 07, France.

Email address: stephane.loisel@univ-lyon1.fr 
Note that the probability $\mathrm{P}\left(I_{T}=0\right)$ is the probability of nonruin within finite time $T . I_{T}$ may be seen as the penalty the company will have to pay due to its insolvency until the time horizon $T$. From an economic point of view, it seems more consistent to consider

$$
I_{g, h}(u)=\int_{0}^{T}\left(\mathbf{1}_{\left\{u+X_{t} \geq 0\right\}} g\left(\left|u+X_{t}\right|\right)-\mathbf{1}_{\left\{u+X_{t} \leq 0\right\}} h\left(\left|u+X_{t}\right|\right)\right) \mathrm{d} t,
$$

with $0 \leq g \leq h$, where $g$ corresponds to a reward function for positive reserves and $h$ is a penalty function in case of insolvency. To be consistent with the theory of utility functions, $g$ should be increasing and concave, and $h$ should be increasing and convex in the classical case. In addition, $g \leq h$ because the cost of ruin is usually higher than the reward at the corresponding wealth level.

These risk measures may be differentiated with respect to the initial reserve $u$, which makes it possible to compute them quite easily as integrals of other functions of $u$, such as the probability of ruin or the total time in the red. Moreover, they have the advantage that the integral over $t$ and the mathematical expectation may be permuted, thanks to Fubini's theorem.

Statements and proofs of our differentiation theorems can be found in Sections 2 and 3. In Section 4, we present examples of applications to one-dimensional risk measures; in particular, a closed-form formula is derived for $\mathrm{E}\left(I_{\infty}\right)$ in the Poisson-exponential case.

We can also use these concepts to construct risk measures for multidimensional risk processes, modelling different lines of business of an insurance company (car insurance, health insurance, etc.). In this framework, determining the global initial reserve needed for the global expected penalty to be sufficiently small requires finding the optimal allocation of this reserve. Differentiation of one-dimensional risk measures is useful for this purpose. These issues are discussed in Section 5.

\section{Differentiation theorems}

Let $(\Omega, \mathcal{F}, \mathrm{P})$ be a probability space. In what follows, for $T \in[0, \infty]$, we will denote the time in the red until time $T$ by

$$
\tau(u, T)=\int_{0}^{T} \mathbf{1}_{\left\{u+X_{t}<0\right\}} \mathrm{d} t .
$$

In most cases, $T$ will be fixed and we will use the notation $\tau(u)$ instead of $\tau(u, T)$. In this section, we assume that $T<\infty$.

Theorem 1. Assume that $T \in \mathbb{R}^{+}$. Let $\left(X_{t}\right)_{t \in[0, T)}$ be a stochastic process with almost surely time-integrable sample paths. For $u \in \mathbb{R}$, denote by $\tau(u)$ the random variable corresponding to the time spent below 0 by the process $u+X_{t}$ between the fixed times 0 and $T$,

$$
\tau(u)=\int_{0}^{T} \mathbf{1}_{\left\{u+X_{t}<0\right\}} \mathrm{d} t
$$

let $\tau_{0}(u)$ represent the time spent at 0 by the process $u+X_{t}$,

$$
\tau_{0}(u)=\int_{0}^{T} \mathbf{1}_{\left\{u+X_{t}=0\right\}} \mathrm{d} t ;
$$

let $I_{T}(u)$ represent the negative part of the process $u+X_{t}$ time-integrated between 0 and $T$,

$$
I_{T}(u)=\int_{0}^{T} \mathbf{1}_{\left\{u+X_{t}<0\right\}}\left|u+X_{t}\right| \mathrm{d} t
$$




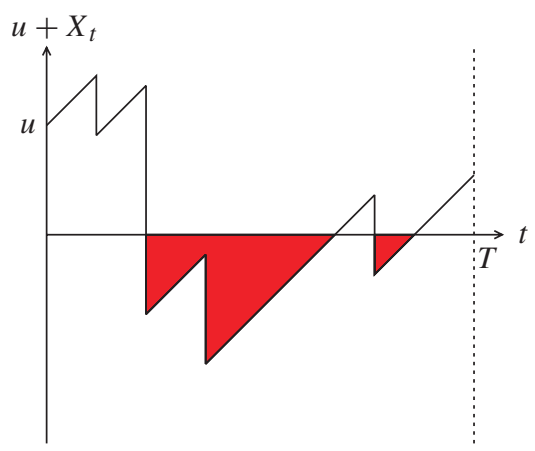

FIGURE 1: The shaded area represents $I_{T}(u)=\int_{0}^{T} \mathbf{1}_{\left\{u+X_{t}<0\right\}}\left|u+X_{t}\right| \mathrm{d} t$.

and let $f(u)=\mathrm{E}\left(I_{T}(u)\right)$. If $\mathrm{E}\left(\tau_{0}(u)\right)=0$ then $f$ is differentiable at $u$, with

$$
f^{\prime}(u)=-\mathrm{E}(\tau(u)) .
$$

$I_{T}(u)$ is illustrated in Figure 1.

Proof. Fix $u \in \mathbb{R}$ and, for $\varepsilon \geq 0$, set

$$
\tau_{\varepsilon}(u)=\int_{0}^{T} \mathbf{1}_{\left\{\left|u+X_{t}\right|<\varepsilon\right\}} \mathrm{d} t .
$$

Here, $\tau_{\varepsilon}(u)$ represents the time spent by the process $u+X_{t}$ in the interval $(-\varepsilon, \varepsilon)$ between time 0 and time $T$.

For each sample path (considered as a function of time $t$ ),

$$
t \mapsto \mathbf{1}_{\left\{\left|u+X_{t}\right|<\varepsilon\right\}}
$$

converges pointwise, from above, to

$$
t \mapsto \mathbf{1}_{\left\{u+X_{t}=0\right\}}
$$

Additionally, the integral of each of the indicator functions is bounded by $T$. From the monotone convergence theorem, $\tau_{\varepsilon}$ is decreasing with respect to $\varepsilon$ and converges to $\tau_{0}$. From the monotone convergence theorem (this time for the expectation), $\mathrm{E}\left(\tau_{\varepsilon}\right) \downarrow \mathrm{E}\left(\tau_{0}\right)$ as $\varepsilon \downarrow 0$ because $\mathrm{E}\left(\tau_{\varepsilon}\right) \leq T$ for all $\varepsilon \geq 0$. To complete the proof, we need the following lemma.

Lemma 1. For $\varepsilon \in \mathbb{R}$,

$$
\left|I_{T}(u+\varepsilon)-I_{T}(u)+\varepsilon \tau(u)\right| \leq|\varepsilon| \tau_{\varepsilon}(u) .
$$

Proof. For $\varepsilon>0$, we have $\left\{u+\varepsilon+X_{t}<0\right\} \subset\left\{u+X_{t}<0\right\}$, whence

$$
\begin{aligned}
I_{T}(u+\varepsilon)-I_{T}(u) \\
\quad=\int_{0}^{T}\left(\left|u+\varepsilon+X_{t}\right|-\left|u+X_{t}\right|\right) \mathbf{1}_{\left\{u+X_{t}<0\right\}} \mathrm{d} t-\int_{0}^{T}\left|u+\varepsilon+X_{t}\right| \mathbf{1}_{\left\{-\varepsilon<u+X_{t}<0\right\}} \mathrm{d} t \\
\quad=-\varepsilon \int_{0}^{T} \mathbf{1}_{\left\{u+X_{t}<0\right\}} \mathrm{d} t-\int_{0}^{T}\left|u+\varepsilon+X_{t}\right| \mathbf{1}_{\left\{-\varepsilon<u+X_{t}<0\right\}} \mathrm{d} t .
\end{aligned}
$$


On the right-hand side of (1), the first term corresponds to $-\varepsilon \tau(u)$, while the absolute value under the integral of the second term is less than $\varepsilon$ on the support of the indicator function it multiplies. Hence,

$$
\left|I_{T}(u+\varepsilon)-I_{T}(u)+\varepsilon \tau(u)\right|<\int_{0}^{T} \varepsilon \mathbf{1}_{\left\{-\varepsilon<u+X_{t}<0\right\}} \mathrm{d} t,
$$

which proves the lemma for $\varepsilon>0$. A symmetrical procedure proves the case in which $\varepsilon \leq 0$.

Now, from Lemma 1 ,

$$
\left|\mathrm{E}\left(I_{T}(u+\varepsilon)\right)-\mathrm{E}\left(I_{T}(u)\right)+\varepsilon \mathrm{E}(\tau(u))\right| \leq|\varepsilon| \mathrm{E}\left(\tau_{\varepsilon}(u)\right)
$$

and

$$
\mathrm{E}\left(I_{T}(u+\varepsilon)\right)=\mathrm{E}\left(I_{T}(u)\right)-\varepsilon \mathrm{E}(\tau(u))+\varepsilon v(u, \varepsilon),
$$

where

$$
|v(u, \varepsilon)| \leq \mathrm{E}\left(\tau_{\varepsilon}(u)\right) \rightarrow \mathrm{E}\left(\tau_{0}(u)\right)=0
$$

as $\varepsilon \rightarrow 0$, which proves that $f$ is differentiable with respect to $u$, with $f^{\prime}(u)=-\mathrm{E}(\tau(u))$ for $u \in \mathbb{R}$.

Corollary 1. Using the notation of Theorem 1, let $X_{t}=c t-S_{t}$, where $S_{t}$ is a jump process such that $S_{t}$ almost surely has a finite number of nonnegative jumps in every finite interval, and such that $X_{t}$ has a positive drift (that is, $X_{t} \rightarrow \infty$ almost surely). Then, for $u \in \mathbb{R}, f$ defined as above is differentiable in $\mathbb{R}$, with $f^{\prime}(u)=-\mathrm{E}(\tau(u))$.

Proof. We need only show that

$$
\mathrm{E}\left(\tau_{0}(u)\right)=\int_{0}^{T} \mathbf{1}_{\left\{u+c t-S_{t}=0\right\}} \mathrm{d} t=0 .
$$

Note that $R_{t}=u+c t-S_{t}$ is a process whose sample paths are almost surely increasing between two consecutive jump times, and that the number of jumps is almost surely finite on the time interval $[0, T]$. Between two times at which the process is 0 , there must be at least one jump time. This implies that the number of visits of the process to 0 is almost surely finite, as it is less than $N_{T}+1$, where $N_{T}$ is the number of jumps between 0 and $T$. Hence, $\mathrm{E}\left(\tau_{0}\right)=0$ and the result follows from Theorem 1 .

Proposition 1. If $N$ is a null subset of $\mathbb{R}^{+}$for the Lebesgue measure, then the condition $\mathrm{E}\left(\tau_{0}\right)=0$ is satisfied by all processes for which the distribution of $R_{t}$ has no atom (point mass) at 0 for any $t \in \mathbb{R}^{+}-N$. Theorem 1 is also satisfied for this wide class of processes.

Proof. For $T \in[0, \infty]$, from Fubini's theorem,

$$
\mathrm{E}\left(\tau_{0}(T)\right) \leq \mathrm{E}\left(\int_{0}^{\infty} \mathbf{1}_{\left\{R_{t}=0\right\}} \mathrm{d} t\right)=\int_{0}^{\infty} \mathrm{P}\left(R_{t}=0\right) \mathrm{d} t,
$$

which provides the required result.

Theorem 2. Let $g \in C^{1}\left(\mathbb{R}^{+}, \mathbb{R}^{+}\right)$be a convex or concave function such that $g(0)=0$, where $C^{1}\left(\mathbb{R}^{+}, \mathbb{R}^{+}\right)$denotes the set of continuously differentiable functions from $\mathbb{R}^{+}$to $\mathbb{R}^{+}$. Let $X_{t}$ be a stochastic process such that, for $u \in \mathbb{R}, t \mapsto \mathbf{1}_{\left\{u+X_{t}<0\right\}} g\left(-\left(u+X_{t}\right)\right)$ is almost surely 
integrable with respect to $t$, and let $I_{g}$ be the function, from $\mathbb{R}$ into the space of nonnegative random variables, defined by

$$
I_{g}(u)=\int_{0}^{T} \mathbf{1}_{\left\{u+X_{t}<0\right\}} g\left(-\left(u+X_{t}\right)\right) \mathrm{d} t
$$

for $u \in \mathbb{R}$. Finally, let $f(\cdot)=\mathrm{E}\left(I_{g}(\cdot)\right)$.

For $u \in \mathbb{R}$, if $f(u)<\infty, \mathrm{E}\left(I_{g^{\prime}}(u)\right)<\infty$, and $\mathrm{E}\left(\tau_{0}(u)\right)=0$, then $f$ is differentiable at point $u$, with

$$
f^{\prime}(u)=-\mathrm{E}\left(\int_{0}^{T} \mathbf{1}_{\left\{u+X_{t}<0\right\}} g^{\prime}\left(\left|u+X_{t}\right|\right) \mathrm{d} t\right) .
$$

Proof. Fix $u \in \mathbb{R}$. We have

$$
\begin{aligned}
\frac{I_{g}(u+\varepsilon)-I_{g}(u)}{\varepsilon}= & \int_{0}^{T} \mathbf{1}_{\left\{u+X_{t}<0\right\}} \frac{g\left(\left|u+\varepsilon+X_{t}\right|\right)-g\left(\left|u+X_{t}\right|\right)}{\varepsilon} \mathrm{d} t \\
& -\int_{0}^{T} \mathbf{1}_{\left\{-\varepsilon<u+X_{t}<0\right\}} \frac{g\left(\left|u+\varepsilon+X_{t}\right|\right)}{\varepsilon} \mathrm{d} t .
\end{aligned}
$$

For $t \in[0, T]$, if $g$ is convex then

$$
\mathbf{1}_{\left\{u+X_{t}<0\right\}} \frac{g\left(-\left(u+\varepsilon+X_{t}\right)\right)-g\left(-\left(u+X_{t}\right)\right)}{-\varepsilon} \uparrow \mathbf{1}_{\left\{u+X_{t}<0\right\}} g^{\prime}\left(-\left(u+X_{t}\right)\right)
$$

almost surely as $\varepsilon \downarrow 0$, from the increase of the rate of increase of $g$, and if $g$ is concave then

$$
\mathbf{1}_{\left\{u+X_{t}<0\right\}} \frac{g\left(-\left(u+\varepsilon+X_{t}\right)\right)-g\left(-\left(u+X_{t}\right)\right)}{-\varepsilon} \downarrow \mathbf{1}_{\left\{u+X_{t}<0\right\}} g^{\prime}\left(-\left(u+X_{t}\right)\right)
$$

almost surely as $\varepsilon \downarrow 0$, from the increase of the rate of decrease of $g$. From the monotone convergence theorem,

$$
\mathrm{E}\left(\mathbf{1}_{\left\{u+X_{t}<0\right\}} \frac{g\left(-\left(u+\varepsilon+X_{t}\right)\right)-g\left(-\left(u+X_{t}\right)\right)}{\varepsilon}\right) \rightarrow-\mathrm{E}\left(\mathbf{1}_{\left\{u+X_{t}<0\right\}} g^{\prime}\left(-\left(u+X_{t}\right)\right)\right)
$$

for $t \in[0, T]$, and, from Fubini's theorem,

$$
\mathrm{E}\left(\int_{0}^{T} \mathbf{1}_{\left\{u+X_{t}<0\right\}} \frac{g\left(-\left(u+\varepsilon+X_{t}\right)\right)-g\left(-\left(u+X_{t}\right)\right)}{\varepsilon} \mathrm{d} t\right) \rightarrow-\mathrm{E}\left(I_{g^{\prime}}(u)\right)
$$

as $\varepsilon \downarrow 0$. Hence,

$$
\left|f(u+\varepsilon)-f(u)+\varepsilon \mathrm{E}\left(I_{g^{\prime}}(u)\right)+\varepsilon w(u, \varepsilon)\right| \leq \mathrm{E}\left(\int_{0}^{T} \mathbf{1}_{\left\{-\varepsilon<u+X_{t}<0\right\}} g\left(-\left(u+\varepsilon+X_{t}\right)\right) \mathrm{d} t\right)
$$

with $w(u, \varepsilon) \rightarrow 0$ as $\varepsilon \downarrow 0$, and

$$
\left|f(u+\varepsilon)-f(u)+\varepsilon \mathrm{E}\left(I_{g^{\prime}}(u)\right)+\varepsilon w(u, \varepsilon)\right| \leq K \varepsilon \mathrm{E}\left(\tau_{\varepsilon}(u)\right),
$$

where $K=\sup _{x \in[0, \varepsilon]}\left|g^{\prime}(x)\right|$. Now,

$$
\mathrm{E}\left(I_{g}(u+\varepsilon)\right)=\mathrm{E}\left(I_{g}(u)\right)-\varepsilon \mathrm{E}\left(I_{g^{\prime}}(u)\right)+\varepsilon(v(u, \varepsilon)-w(u, \varepsilon)),
$$


where

$$
|v(u, \varepsilon)| \leq K \mathrm{E}\left(\tau_{\varepsilon}(u)\right) \rightarrow K \mathrm{E}\left(\tau_{0}(u)\right)=0
$$

as $\varepsilon \downarrow 0$, which proves that $f$ is right-differentiable at $u$, with

$$
f_{\mathrm{r}}^{\prime}(u)=-\mathrm{E}\left(\int_{0}^{T} \mathbf{1}_{\left\{u+X_{t}<0\right\}} g^{\prime}\left(-\left(u+X_{t}\right)\right) \mathrm{d} t\right) .
$$

By similar reasoning, $f$ can be shown to be left-differentiable, with $f_{1}^{\prime}=f_{\mathrm{r}}^{\prime}$, which ends the proof.

\section{Differentiation of the average time in the red, and other generalizations}

Recall that the time in the red is the time spent below 0 by the wealth process between time 0 and some fixed time horizon $T$ :

$$
\tau(u)=\int_{0}^{T} \mathbf{1}_{\left\{u+X_{t}<0\right\}} \mathrm{d} t .
$$

Let us first suppose that $T$ is finite.

Theorem 3. Let $X_{t}=c t-S_{t}$, where $S_{t}$ is a jump process satisfying the following hypothesis.

(H1) $S_{t}$ has a finite expected number of nonnegative jumps in every finite interval and, for each $t$, the distribution of $S_{t}$ has no atom on $(0, \infty)$.

For example, $S_{t}$ might be a compound Poisson process with a continuous jump size distribution. Let $T<\infty$ and define $h$ by $h(u)=\mathrm{E}(\tau(u))$ for $u \in \mathbb{R}$. The function $h$ is differentiable in $\mathbb{R}_{*}^{+}=(0, \infty)$, with

$$
h^{\prime}(u)=-\frac{1}{c} \mathrm{E}\left(N^{0}(u, T)\right)
$$

for $u>0$, where $N^{0}(u, T)=\operatorname{card}\left(\left\{t \in[0, T]: u+c t-S_{t}=0\right\}\right)$.

Proof. The number of jumps $N_{T}$ and, hence, $N^{0}(u, T)$ are finite almost surely. Consider a sample path $\left(X_{t}(\omega)\right)_{0 \leq t \leq T}$, for $\omega \in \Omega$. Let $R_{t}=u+X_{t}$ and denote by $T_{i}$ the $i$ th jump time. Define

$$
\varepsilon_{0}(\omega)=\inf _{n \leq N_{T}, R_{T_{n}}>0} R_{T_{n}}
$$

If $N^{0}(u, T)=0$ then define

$$
\varepsilon^{+}=\inf \left(\left\{u+X_{t}, 0 \leq t \leq T\right\} \cap \mathbb{R}^{+}\right)
$$

and

$$
\varepsilon^{-}=-\sup \left(\left\{u+X_{t}, 0 \leq t \leq T\right\} \cap \mathbb{R}^{-}\right) .
$$

Here, $\varepsilon^{-}$and $\varepsilon^{+}$are almost surely positive. If $|\varepsilon|<\inf \left\{\varepsilon^{+}, \varepsilon^{-}\right\}$then $\tau(u-\varepsilon)-\tau(u)=0$, and the remainder of the proof remains valid. If $N^{0}(u, T) \neq 0$ then denote by $t_{i}, 1 \leq i \leq N^{0}(u, T)$, the time of the $i$ th visit of $R_{t}$ to 0 , and by $t_{i}^{\prime}$ the time of the first jump of $R_{t}$ after $t_{i}$. The sample paths of the process $R_{t}$ are almost surely right-continuous, and the probability that $R_{T}=0$ is zero. Therefore, we may consider

$$
\varepsilon_{1}(\omega)=\min \left\{\min _{1 \leq i \leq N^{0}(u, T)} c\left(t_{i}^{\prime}-t_{i}\right), c\left(T-t_{N^{0}(u, T)}\right)\right\} .
$$


Then, for $0<\varepsilon<\min \left\{\varepsilon_{0}(\omega), \varepsilon_{1}(\omega)\right\}$,

$$
\left\{0<u+c t-S_{t}<\varepsilon\right\}=\bigcup_{i=1}^{N^{0}(u, T)}\left\{\left(t_{i}, t_{i}+\frac{\varepsilon}{c}\right)\right\}
$$

and, so,

$$
\begin{aligned}
\tau(u-\varepsilon)-\tau(u) & =\int_{0}^{T} \mathbf{1}_{\left\{u-\varepsilon+c t-S_{t}<0\right\}} \mathrm{d} t-\int_{0}^{T} \mathbf{1}_{\left\{u+c t-S_{t}<0\right\}} \mathrm{d} t \\
& =\int_{0}^{T} \mathbf{1}_{\left\{0 \leq u+c t-S_{t}<\varepsilon\right\}} \mathrm{d} t \\
& =\sum_{k=1}^{N^{0}(u, T)} \frac{\varepsilon}{c} .
\end{aligned}
$$

Hence,

$$
\frac{\tau(u-\varepsilon)-\tau(u)}{\varepsilon} \rightarrow \frac{1}{c} N^{0}(u, T)
$$

almost surely as $\varepsilon \rightarrow 0$. Moreover, between two consecutive jumps of $R_{t}$, the difference between the two integrals corresponding to those on the right-hand side of (2) is less than $\varepsilon / c$ in absolute value, whence

$$
\int_{T_{i}}^{T_{i+1}} \mathbf{1}_{\left\{0 \leq u+c t-S_{t}<\varepsilon\right\}} \mathrm{d} t \leq \frac{\varepsilon}{c} .
$$

So, for $\varepsilon>0$ sufficiently small, and writing $T_{N_{T}+1}=T$ and $T_{0}=0$, we have

$$
\begin{aligned}
\frac{\tau(u-\varepsilon)-\tau(u)}{\varepsilon} & =\sum_{i=0}^{N_{T}} \frac{1}{\varepsilon} \int_{T_{i}}^{T_{i+1}} \mathbf{1}_{\left\{0 \leq u+c t-S_{t}<\varepsilon\right\}} \mathrm{d} t \\
& \leq \sum_{i=0}^{N_{T}} \frac{1}{\varepsilon} \frac{\varepsilon}{c} \\
& \leq \frac{1}{c}\left(N_{T}+1\right) .
\end{aligned}
$$

Hence, from the dominated convergence theorem,

$$
\mathrm{E}\left(\frac{\tau(u-\varepsilon)-\tau(u)}{\varepsilon}\right) \rightarrow \frac{1}{c} \mathrm{E}\left(N^{0}(u, T)\right)
$$

as $\varepsilon \rightarrow 0$. This proves that $h$ is left-differentiable in $\mathbb{R}_{*}^{+}$, with

$$
h_{1}^{\prime}(u)=-\frac{1}{c} \mathrm{E}\left(N^{0}(u, T)\right)
$$

for $u>0$.

By similar reasoning, $h$ is right-differentiable in $\mathbb{R}_{*}^{+}$, with $h_{1}^{\prime}=h_{\mathrm{r}}^{\prime}$. Hence, $h$ is differentiable in $\mathbb{R}_{*}^{+}$, with $h^{\prime}(u)=-(1 / c) \mathrm{E}\left(N^{0}(u, T)\right)$ for $u>0$.

Remark 1. This provides the second-order derivative of $\mathrm{E}\left(I_{T}(\cdot)\right)$, which appears to be positive. Thus, $\mathrm{E}\left(I_{T}(\cdot)\right)$ is strictly convex, which will be very important for the minimization in Section 5. 
Remark 2. This second-order derivative corresponds, in the general case, to the expectation of the local time $L_{T}(0)$ spent at 0 by the process $u+X_{t}$, up to time T:

$$
L_{T}(0)=\lim _{\varepsilon \downarrow 0}\left(\frac{1}{2 \varepsilon} \int_{0}^{T} \mathbf{1}_{\left\{\left|u+X_{t}\right|<\varepsilon\right\}} \mathrm{d} t\right) .
$$

Theorem 4. Let $g$ and $h$ be two convex or concave functions in $C^{1}\left(\mathbb{R}^{+}, \mathbb{R}^{+}\right)$, such that $g(x) \geq$ $g(0)$ and $h(x) \geq h(0)$ for $x \geq 0$. Let $X_{t}$ be a stochastic process such that $t \mapsto g\left(u+X_{t}\right)$ and $t \mapsto h\left(u+X_{t}\right)$ are almost surely integrable on $[0, T]$. Let $I_{g}^{+}$be the function, from $\mathbb{R}$ into the space of nonnegative random variables, defined by

$$
I_{g}^{+}(u)=\int_{0}^{T} \mathbf{1}_{\left\{u+X_{t} \geq 0\right\}} g\left(u+X_{t}\right) \mathrm{d} t
$$

for $u \geq 0$, and let $f(\cdot)=\mathrm{E}\left(I_{g}^{+}(\cdot)\right)-\mathrm{E}\left(I_{h}(\cdot)\right)$.

If, for $u \in \mathbb{R}$,

$$
\mathrm{E}\left(I_{g}^{+}(u)\right)<\infty, \quad \mathrm{E}\left(I_{h}(u)\right)<\infty, \quad \mathrm{E}\left(I_{g^{\prime}}^{+}(u)\right)<\infty, \quad \mathrm{E}\left(I_{h^{\prime}}(u)\right)<\infty,
$$

and $\mathrm{E}\left(\tau_{0}(u)\right)=0$, then $f$ is differentiable in $\mathbb{R}_{*}^{+}$, with

$$
f^{\prime}(u)=\mathrm{E}\left(I_{g^{\prime}}^{+}(u)\right)-\mathrm{E}\left(I_{h^{\prime}}(u)\right)-(g(0)+h(0)) \mathrm{E}\left(L_{T}(0)\right)
$$

for $u>0$.

Corollary 2. If, in addition to the hypotheses of Theorem 4, we assume that $X_{t}=c t-S_{t}$, where $S_{t}$ satisfies hypothesis (H1) of Theorem 3, then $f^{\prime}(u)$ may be rewritten, for $u>0$, as

$$
f^{\prime}(u)=\mathrm{E}\left(I_{g^{\prime}}^{+}(u)\right)-\mathrm{E}\left(I_{h^{\prime}}(u)\right)+\frac{(g(0)+h(0)) \mathrm{E}\left(N^{0}(u, T)\right)}{c},
$$

where $N^{0}(u, T)$ is as defined in Theorem 3.

Proof. The proof follows immediately from Theorem 4, after replacing the last term in (3) using the proof of Theorem 3 .

Proof of Theorem 4. We write

$$
I_{g}^{+}(u)-I_{h}(u)=-\tilde{I}_{(g-g(0))}(-u)-I_{(h-h(0))}(u)-h(0) \tau(u)+g(0)(T-\tau(u)),
$$

where $\tilde{I}_{g}$ is obtained from $I_{g}$ by changing $X_{t}$ into $-X_{t}$. From the linearity of expectations and differentiation, applying Theorem 2 to $g-g(0)$, with $-X_{t}$ in place of $X_{t}$, and to $h-h(0)$, with $X_{t}$ unchanged, and using Theorem 3 completes the proof of Theorem 4 .

Theorem 5. Theorem 1 remains valid with $T=\infty$ if, in addition to the assumptions made there, the process $X_{t}$ converges almost surely to $\infty$ as $t \rightarrow \infty$, and $\mathrm{E}\left(I_{\infty}\right)<\infty$ and $\mathrm{E}(\tau(u, \infty))<\infty$ for $u \geq 0$.

Proof. The proof relies on the same kind of reasoning as was used in the proof of Theorem 1.

Remark 3. These conditions of integrability are fulfilled if the time spent below 0 during a single period of ruin is integrable. 
We denote by $\psi(u)$ the probability of ruin in infinite time, with initial reserve $u$.

Theorem 6. Theorem 3 remains valid with $T=\infty$ if, in addition to the assumptions made there, $X_{t}$ has a positive drift and $\tau(u)$ is integrable for all $u>0$. Furthermore, in the compound Poisson case,

$$
h^{\prime}(u)=-\frac{1}{c} \frac{1}{1-\psi(0)} \psi(u)
$$

for $u>0$.

Proof. For $T \in[0, \infty]$, recall the notation

$$
\tau(u, T)=\int_{0}^{T} \mathbf{1}_{\left\{u+X_{t}<0\right\}} \mathrm{d} t .
$$

Note that $\left(N^{0}(u, n)\right)_{n \geq 0}$ is a nondecreasing sequence of random variables that converges surely to $N^{0}(u, \infty)$, which is possibly infinite. We shall show that $\mathrm{E}\left(N^{0}(u, \infty)\right)<\infty$.

As $t \rightarrow \infty, u+X_{t} \rightarrow \infty$ almost surely. Hence, almost surely, $N^{0}(u, \infty)<\infty$ and is equal to the number of periods of ruin:

$$
N^{0}(u, \infty)=\operatorname{card}\left(\left\{t>0: u+c t-S_{t}<0 \text { and } u+c t^{-}-S_{t^{-}}>0\right\}\right) .
$$

Indeed, after each period of ruin there is a recovery because $X_{t}$ converges almost surely to $\infty$ as $t$ goes to $\infty$, and the number of jumps that lead exactly to the value 0 is almost surely finite. Furthermore, in the compound Poisson case, the number of periods of ruin has the distribution

$$
\mathrm{P}\left(N^{0}(u, \infty)=n\right)=\psi(u) \psi(0)^{n-1}(1-\psi(0))
$$

for $n \geq 1$, with $\mathrm{P}\left(N^{0}(u, \infty)=0\right)=1-\psi(u)$. Hence, $N^{0}(u, \infty)$ follows a zero-modified geometric distribution, i.e. $\mathrm{P}\left(N^{0}(u, \infty)=0\right)=1-\psi(u)$ and, for $n>0$,

$$
\mathrm{P}\left(N^{0}(u, \infty)=n \mid N^{0}(u, \infty)>0\right)=\psi(0)^{n-1}(1-\psi(0)) .
$$

Hence, $N^{0}(u, \infty)$ is integrable and

$$
\mathrm{E}\left(N^{0}(u, \infty)\right)=\psi(u) \frac{1}{1-\psi(0)} .
$$

For all $\omega$ and for $\varepsilon>0$, the function

$$
(T, \omega) \mapsto \frac{\tau(u+\varepsilon, T)-\tau(u, T)}{\varepsilon} \omega
$$

is increasing with respect to $T$, and the limit of its expectation, as $\varepsilon \downarrow 0$, is $-(1 / c) \mathrm{E}\left(N^{0}(u, T)\right)$. From the monotone convergence theorem,

$$
\mathrm{E}\left(\lim _{\varepsilon \downarrow 0}\left(\frac{\tau(u+\varepsilon, \infty)-\tau(u, \infty)}{\varepsilon}\right)\right)=-\frac{1}{c} \mathrm{E}\left(N^{0}(u, \infty)\right) .
$$

Remark 4. In infinite time, the probability of ruin may be regarded (up to multiplication by a constant) as the expectation of the local time spent by the process at 0 . 


\section{Applications in the one-dimensional case}

Theorem 7. In the Poisson $(\lambda)-\operatorname{Exp}(1 / \mu)$ case, with positive safety loading $\rho=(c-\lambda \mu) / \lambda \mu$, we have $\psi(u)=(1-\mu R) \mathrm{e}^{-R u}$, where $R=(1 / \mu)(1-\lambda \mu / c)$. Hence, for $T=\infty$,

$$
\mathrm{E}(\tau(u))=\frac{1-\mu R}{c \mu R^{2}} \mathrm{e}^{-R u}
$$

and

$$
\mathrm{E}\left(I_{\infty}(u)\right)=\frac{1-\mu R}{c \mu R^{3}} \mathrm{e}^{-R u} .
$$

Proof. The proof follows simply from integration of the well-known formula for $\psi(u)$, as the functions considered tend to 0 as $u \rightarrow \infty$.

This method provides a way of determining the average total time in the red from the integral of the probability of ruin. Dos Reis (1993) derived this result for $\mathrm{E}(\tau(u, \infty))$ by considering the number of times ruin occurs, and using the distributions of the lengths of the first and subsequent periods in the red, the latter of which were derived by Gerber (1988).

Remark 5. It is possible to derive $\mathrm{E}\left(I_{\infty}(u)\right)$ for gamma-distributed or phase-type-distributed claim amounts, as we know the probability of ruin in these cases. (For brevity, we do not report the results here.)

The parallel with the Brownian case is also interesting. The local time at $x$ of a standard Brownian motion $W_{t}$ is defined by

$$
L_{t}(x)=\lim _{\varepsilon \downarrow 0} \frac{1}{4 \varepsilon} \int_{0}^{t} \mathbf{1}_{\left\{\left|W_{s}-x\right|<\varepsilon\right\}} \mathrm{d} s .
$$

This provides a density for the occupation time $\Gamma_{t}(B)$ of a Borel set $B$, between 0 and $t$ :

$$
\Gamma_{t}(B)=\int_{B} 2 L_{t}(x) \mathrm{d} x
$$

Theorem 8. (Lévy's Brownian local-time representation theorem with downcrossings.) Let $D_{t}(\varepsilon)$ be the number of downcrossings of the interval $[0, \varepsilon]$ by the process $W_{s}$, between 0 and t. Then, $2 L_{t}(0)=\lim _{\varepsilon \downarrow 0} \varepsilon D_{t}(\varepsilon)$.

This well-known theorem can be viewed as a limiting case of Theorem 3 .

\section{Multidimensional risk measures and optimal allocation}

For a one-dimensional risk process, one classical goal is to determine the minimal initial reserve $u_{\varepsilon}$ needed for the probability of ruin to be less than $\varepsilon$. In a multidimensional framework, modelling the evolution of the different lines of business of an insurance company as a multirisk process $\left(u_{1}+X_{t}^{1}, \ldots, u_{K}+X_{t}^{K}\right)$ (where $u_{k}+X_{t}^{k}$ corresponds to the wealth of the $k$ th line of business at time $t$ ), we might seek the global initial reserve $u$ which ensures that the probability of ruin $\psi$ satisfies $\psi\left(u_{1}, \ldots, u_{K}\right) \leq \varepsilon$ for the optimal allocation $\left(u_{1}, \ldots, u_{K}\right)$ such that

$$
\psi\left(u_{1}, \ldots, u_{K}\right)=\inf _{v_{1}+\cdots+v_{K}=u} \psi\left(v_{1}, \ldots, v_{K}\right),
$$

with

$$
\psi\left(u_{1}, \ldots, u_{K}\right)=\mathrm{P}\left(\text { there exist a } k \in[1, K] \text { and a } t>0 \text { such that } u_{k}+X_{t}^{k}<0\right) .
$$


Instead of minimizing the probability of crossing certain barriers, it may be more interesting to minimize the sum of the expected costs of ruin, until time $T$, of each line of business. This may be represented by the expectation of the sum of integrals over time of the negative part of the process. In both cases, finding the necessary global reserve requires determination of the optimal allocation. In the previous sections, we have shown how to compute $\mathrm{E}\left(I_{T}\right)$ for one line of business, and the linearity of the expectation makes it possible to compute the sum for $K$ dependent lines of business just as in the independent case; the structure of dependence between lines of business has no impact on this risk measure. This may be considered to be a problem of optimal allocation of resources under budget constraints, as in economics, the goal being to maximize the utility function given by the sum of the $-\mathrm{E}\left(I_{T}^{i}\right)$, where $I_{T}^{i}, i=1, \ldots, K$, is the penalty paid by the company for the $i$ th line of business.

\subsection{Minimizing the penalty function}

Recall that what has to be minimized is

$$
A\left(u_{1}, \ldots, u_{K}\right)=\sum_{k=1}^{K} \mathrm{E}\left(I_{T}^{k}\right)
$$

where

$$
\mathrm{E}\left(I_{T}^{k}\right)=\mathrm{E}\left(\int_{0}^{T}\left|R_{t}^{k}\right| \mathbf{1}_{\left\{R_{t}^{k}<0\right\}} \mathrm{d} t\right)
$$

with $R_{t}^{k}=u_{k}+X_{t}^{k}$ under the constraint $u_{1}+\cdots+u_{K}=u$. Owing to the linearity of the expectation, this is not affected by how the lines of business depend on one another. Denote by $v_{k}\left(u_{k}\right)$ the derivative of $\mathrm{E}\left(I_{T}^{k}\right)$ with respect to $u_{k}$. Using Lagrange multipliers, we can show that if $\left(u_{1}, \ldots, u_{K}\right)$ minimizes $A$, then $v_{k}\left(u_{k}\right)=v_{1}\left(u_{1}\right)$ for all $1 \leq k \leq K$. Computing $v_{k}\left(u_{k}\right)$, we obtain

$$
v_{k}\left(u_{k}\right)=\left(\mathrm{E}\left(\int_{0}^{T}\left|R_{t}^{k}\right| \mathbf{1}_{\left\{R_{t}^{k}<0\right\}} \mathrm{d} t\right)\right)^{\prime}=-\mathrm{E}\left(\tau^{k}\right)=-\int_{0}^{T} \mathrm{P}\left(R_{t}^{k}<0\right) \mathrm{d} t,
$$

where $\tau^{k}$ represents the time spent in the red, between time 0 and time $T$, by line of business $k$. Theorem 1 justifies this statement. The sum of the average times spent under 0 is a decreasing function of the $u_{k}$, so $A$ is strictly convex. On the compact space

$$
s=\left\{\left(u_{1}, \ldots, u_{K}\right) \in\left(\mathbb{R}^{+}\right)^{K}: u_{1}+\cdots+u_{K}=u\right\},
$$

$A$ admits a unique minimum. Thus, the optimal allocation is as follows: there is a subset $J \subset[1, K]$ such that, for $k \notin J$, we have $u_{k}=0$ and, for $k, j \in J$, we have $\mathrm{E}\left(\tau_{k}\right)=\mathrm{E}\left(\tau_{j}\right)$. The interpretation is quite intuitive: the safest lines of business do not require any reserve, and the others share the global reserve in such a way as to have equal average times in the red.

If we relax nonnegativity then, on $\left\{u_{1}+\cdots+u_{K}=u\right\}$, if $\left(u_{1}, \ldots, u_{K}\right)$ is an extremum point for $A$, the average times spent under 0 by the $K$ lines of business are equal to one another. If $\left(u_{1}, \ldots, u_{K}\right)$ is a minimum for the sum of the times spent below 0 by each line of business, then the average number of visits by line of business $k$ is proportional to $c_{k}$, the corresponding premium income rate, and, at infinite time, the ruin probabilities are in fixed proportions. However, the existence of a minimum is not guaranteed, because $\left(u_{1}, \ldots, u_{K}\right)$ is no longer compact. The problem would be more tractable if we minimized the sum of the average times in the red or minimized on the $c_{k}$, because some factors penalize very negative $u_{k}$ in these cases. 


\subsection{Example}

In the Poisson $(\lambda)-\operatorname{Exp}(1 / \mu)$ case, recall that

$$
\mathrm{E}\left(I_{\infty}(u)\right)=\frac{(1-\mu R)}{c \mu R^{3}} \mathrm{e}^{-R u} .
$$

Consider a model with two lines of business, with the following parameters: $\mu_{1}=\mu_{2}=1$, $c_{1}=c_{2}=1, R_{2}=0.4$, and $u=10$. We want to minimize $A\left(u_{1}, u_{2}\right)$ for $0 \leq u_{1}, u_{2} \leq 10$ such that $u_{1}+u_{2}=10$. A slight modification of the adjustment coefficient $R_{1}$ makes the optimal allocation vary strongly. When $R_{1}=0.5>R_{2}$, (see Figure 2), line of business 1 is safer than line 2 , from comparison of the adjustment coefficients, and line 2 should receive a greater initial reserve than line 1 . The optimal allocation is about $\left(u_{1}=3.745990378, u_{2}=6.254009622\right)$. When $R_{1}=0.3<R_{2}$ (see Figure 3), line of business 1 is riskier and, so, should receive a greater initial reserve than line 2 . The optimal allocation in this case is $\left(u_{1}=6.756449750\right.$, $u_{2}=3.243550250$ ). When $R_{1}=0.08$ (see Figure 4 ), the optimal allocation is $\left(u_{1}=10\right.$, $u_{2}=0$ ). In this case, line of business 1 is much more risky than line of business 2 , which justifies the transfer of the entire global initial reserve $u$ to line of business 1 . For more properties or examples of optimal reserve allocation, the interested reader can consult Loisel (2005).

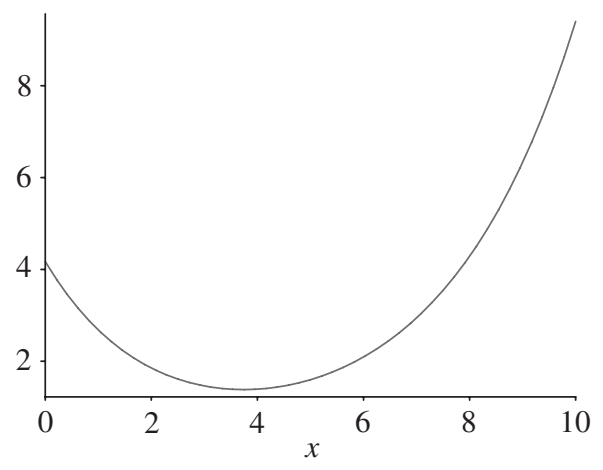

Figure 2: A graph of $A(x, 10-x)$ with $R_{1}=0.5$. Line of business 2 should have greater initial reserve than line 1 .

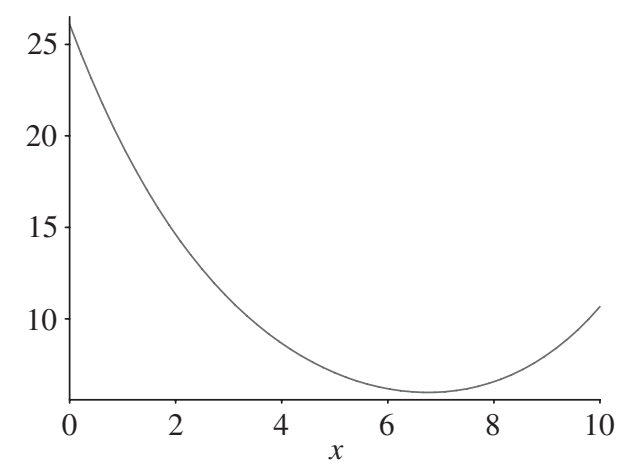

Figure 3: A graph of $A(x, 10-x)$ with $R_{1}=0.3$. Line of business 1 should have greater initial reserve than line 2 . 


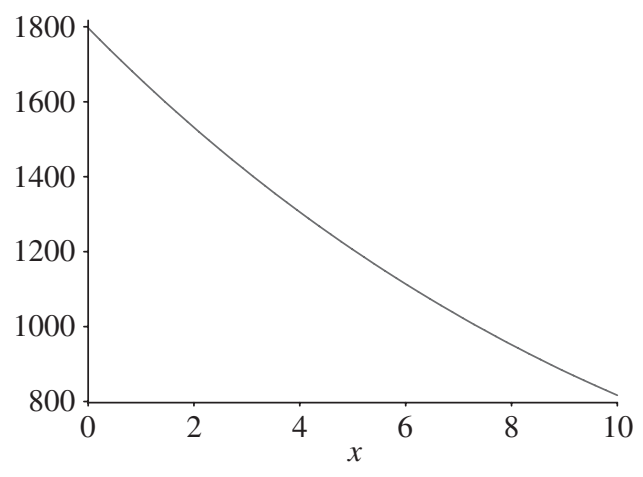

FIGURE 4: A graph of $A(x, 10-x)$ with $R_{1}=0.08$. Line of business 1 should receive the entire initial reserve.

\subsection{Further applications}

The multidimensional risk measure $A$, which does not depend on the structure of dependence between lines of business, is one example of what can be considered. Another possibility would be to minimize the sum $B=\sum_{k=1}^{K} \mathrm{E}\left(\tau_{k}^{\prime}(u)\right)$, where

$$
\mathrm{E}\left(\tau_{k}^{\prime}(u)\right)=\mathrm{E}\left(\int_{0}^{T} \mathbf{1}_{\left\{R_{t}^{k}<0\right\}} \mathbf{1}_{\left\{\sum_{j=1}^{K} R_{t}^{j}>0\right\}} \mathrm{d} t\right) .
$$

Here, $B$ takes dependence into account, and the following proposition shows what can be done.

Proposition 2. Let $X_{t}=c t-S_{t}$, where $S_{t}$ satisfies hypothesis (H1) of Theorem 3, and define $B$ by $B\left(u_{1}, \ldots, u_{K}\right)=\sum_{k=1}^{K} \mathrm{E}\left(\tau_{k}^{\prime}(u)\right)$, for $u \in \mathbb{R}^{K}$. Hence, $B$ is differentiable on $\left(\mathbb{R}_{*}^{+}\right)^{K}$, with

$$
\frac{\partial B}{\partial u_{k}}=-\frac{1}{c_{k}} \mathrm{E}\left(N_{k}^{0}(u, T)\right)
$$

for $u_{1}, \ldots, u_{K}>0$, where $N_{k}^{0}(u, T)=\operatorname{card}\left(\left\{t \in[0, T]: R_{t}^{k}=0\right.\right.$ and $\left.\left.\sum_{j=1}^{K} R_{t}^{j}>0\right\}\right)$.

It is also possible to differentiate with respect to the $c_{k}$ instead of the $u_{k}$.

Theorem 9. Using the notation of Theorem 1, consider the case $X_{t}=c t-S_{t}$, where $S_{t}$ satisfies hypothesis (H1) of Theorem 3, and define $\tilde{f}(c)=\mathrm{E}\left(I_{T}(c)\right)$. If $\mathrm{E}\left(\tau_{0}(c)\right)=0$ for all $c$ then $\tilde{f}$ is differentiable in $\mathbb{R}$, with

$$
\tilde{f}^{\prime}(u)=-\int_{0}^{T} t \mathrm{P}\left(u+c t-S_{t}<0\right) \mathrm{d} t
$$

for $c \in \mathbb{R}$.

It is interesting to look for the optimal allocation of the global premium $c=c_{1}+\cdots+c_{K}$ because, if $c_{k}$ is small enough to make the safety loading negative, the process $R_{t}^{k}$ tends to $-\infty$. Quite often, optimizing with the $c_{k}$ will be easier than with the $u_{k}$ for this reason. These examples illustrate how these differentiation results may be used.

The differentiation results developed here are quite general and may be useful in solving many problems involving multirisk models. For a discussion about multidimensional risk measures, optimal allocation procedures, and the effect of dependence between lines of business, the interested reader can consult Loisel (2005). 


\section{Acknowledgements}

The author is very grateful to Esther Frostig, Christian Mazza, Philippe Picard, Daniel Serant, and the referee for their useful comments and suggestions.

\section{References}

Dos ReIs, A. E. (1993). How long is the surplus below zero? Insurance Math. Econom. 12, 23-38.

Dufresne, F. AND Gerber, H. U. (1988). The surpluses immediately before and at ruin, and the amount of the claim causing ruin. Insurance Math. Econom. 7, 193-199.

Gerber, H. U. (1988). Mathematical fun with ruin theory. Insurance Math. Econom. 7, 15-23.

LoISEL, S. (2005). Finite-time ruin probabilities in the Markov-modulated multivariate compound Poisson model with common shocks, and impact of dependence. Working paper, Cahiers de recherche de l'ISFA, WP2026.

PICARD, P. (1994). On some measures of the severity of ruin in the classical Poisson model. Insurance Math. Econom. 14, 107-115. 